\title{
Performance Analysis of a Single Server Retrial Queueing System with Bernoulli and Phase Type Vacations
}

\author{
J.Ebenesar Anna Bagyam \\ Assistant Professor, Department of Mathematics \\ SNS College of Technology, Coimbatore, \\ Tamil Nadu, India
}

\author{
K. Udaya Chandrika \\ Professor \\ Avinashilingam Deemed University For Women, \\ Coimbatore, Tamil Nadu, India
}

\begin{abstract}
This paper analyses the steady state behavior of an M/G/1 retrial queueing system with Bernoulli and phase type vacations. Customers arrive one by one at the system in a Poisson stream. At the arrival epoch, if the server is busy then the arriving customer joins the orbit. If the server is free, then the arriving customer starts its service immediately. The service time of a customer is assumed to be general. At each service completion epoch, the server may opt to take a phase 1 vacation with probability $\mathrm{p}$ or else with probability $1-\mathrm{p}$ stay in the system for the next service. After the completion of phase 1 vacation the server may take phase 2 vacation with probability q or return back to the system with probability 1-q. The vacation times are assumed to be general. The service times and vacation times are independent of each other. Generating function technique is applied to obtain the system size and orbit size. Numerical examples are provided to illustrate the sensitivity of the performance measures for changes in the parametric of the system.
\end{abstract}

\section{Mathematics Subject Classification}

$60 \mathrm{~K} 25,90 \mathrm{~B} 22$

\section{Keywords}

Retrial queue, Bernoulli vacation, Phase type vacation, Stability condition, Steady state distribution.

\section{INTRODUCTION}

Retrial queueing system is characterized by the feature that the arriving customers who find the server busy join the retrial queue to try again for their requests or leave the service area immediately. These models have been successfully used to many problems in telephone switching systems, telecommunication networks and computer networks. For recent bibliography on retrial queue see $[1,2,10,11]$.

Vacation queues are very important class of queues in real life. Single server queueing models with vacation have been well studied due to their wide application in flexible manufacturing or computer communication systems over more than two decades. Several excellent surveys on single server vacation models have been done by $[4,5,7,9,12]$. Only few authors $[3,6]$ have considering phase type vacation. In this paper a single server retrial queueing system based on Bernoulli schedule and phase type vacation is considered.

\section{MODEL DESCRIPTION}

Consider a single server retrial queue in which customers arrive at the system according to a Poisson process with rate $\lambda$. If an arriving customer finds the server idle, it may obtain service immediately. If the server is found busy or on vacation, the arriving primary customer joins a retrial queue and makes a retrial at a later time. The retrial time is generally distributed with distribution function $\mathrm{A}(\mathrm{x})$, density function $\mathrm{a}(\mathrm{x})$, Laplace Steltjes transform $\mathrm{A} *(\mathrm{~s})$ and the conditional completion rate $\eta(x)=a(x) /(1-A(x))$.

The service times are independent and identically distributed with common distribution function $\mathrm{B}(\mathrm{x})$, density function $\mathrm{b}(\mathrm{x})$,Laplace Steltjes transform $\mathrm{B} *(\mathrm{~s})$, the conditional completion rate $\mu(x)=b(x) /(1-B(x))$ and first two moments are $\mu_{1}$ and $\mu_{2}$

The vacation period of the server has two heterogeneous phases. At each service completion epoch, the server may take a phase 1 vacation with probability $p$ or else continue to be in the system for the next service with probability 1-p. Phase 2 vacation follows the phase 1 vacation in such a way that the server may take phase 2 with probability q or return back to the system with probability 1-q. The distribution function, density function and Laplace Steltjes transform of $i^{\text {th }}$ phase ( $i$ $=1,2)$, vacation times are $\mathrm{V}_{\mathrm{i}}(\mathrm{x}), \mathrm{v}_{\mathrm{i}}(\mathrm{x})$ and $\mathrm{V}_{\mathrm{i}}{ }^{*}(\mathrm{~s})$ respectively. The first two moments are $\beta_{\mathrm{i} 1}$ and $\beta_{\mathrm{i} 2}$ and hazard rate function are $\beta_{\mathrm{i}}(\mathrm{x}), \mathrm{i}=1,2$.

Assume that various stochastic processes involved in the system are independent of each other. At time $t$, let $N(t)$ be the number of customers in the retrial queue. $\mathrm{U}(\mathrm{t})$ the elapsed retrial time of the customer in the retrial queue. $\mathrm{X}(\mathrm{t})$ the elapsed service time of the customer in service. Yi(t) the elapsed $i^{\text {th }}$ phase vacation time $(i=1,2)$.

Let $\mathrm{I}_{0}(\mathrm{t})$ be the probability that at time $\mathrm{t}$, there is no customer in the retrial orbit, the server is idle.

$\mathrm{I}_{\mathrm{n}}(\mathrm{t}, \mathrm{u}) \mathrm{du}, \mathrm{n} \geq 1$ be the joint probability that at time $\mathrm{t}$, there are $\mathrm{n}$ customer in the retrial orbit, the server is idle, and the elapsed retrial time of a customer is between $u$ and $u+d u$.

$W_{n}(t, x) d x, n \geq 0$ be the joint probability that at time $t$, there are $\mathrm{n}$ customer in the retrial orbit and a customer is in service with the elapsed service time between $\mathrm{x}$ and $\mathrm{x}+\mathrm{dx}$.

$\mathrm{V}_{\mathrm{i}, \mathrm{n}}(\mathrm{t}, \mathrm{y}) \mathrm{dy}, \mathrm{n} \geq 0 ; \mathrm{i}=1,2$ be the joint probability that at time $\mathrm{t}$, there are $\mathrm{n}$ customer in the retrial orbit with elapsed vacation time of phase $\mathrm{i}$ between $\mathrm{y}$ and $\mathrm{y}+\mathrm{dy}$. 


\section{STABILITY CONDITION}

In this section necessary and sufficient condition for the system to be stable is derived.

\section{Theorem 1}

The inequality $\lambda\left(\mu_{1}+\mathrm{p} \beta_{11}+\mathrm{pq} \beta_{21}\right)<\mathrm{A}^{*}(\lambda)$ is a necessary and sufficient condition for the system to be stable.

\section{Proof:}

Let $\mathrm{E}(\mathrm{s})$ and $\mathrm{E}(\mathrm{I})$ denote the expected blocked time and expected idle time of the server. From the description of the model $\mathrm{E}(\mathrm{s})=\mu_{1}+\mathrm{p} \beta_{11}+\mathrm{pq} \beta_{21}$.

$\mathrm{P}(\mathrm{I})$, the probability that the server is idle $=\frac{\mathrm{E}(\mathrm{I})}{\mathrm{E}(\mathrm{I})+\mathrm{E}(\mathrm{s})}$

$\mathrm{P}(\mathrm{s})$, the probability that the server is blocked $=\frac{\mathrm{E}(\mathrm{s})}{\mathrm{E}(\mathrm{I})+\mathrm{E}(\mathrm{s})}$.

Exit rate from the retrial queue by entering service is $\mathrm{P}(\mathrm{I}) \frac{\mathrm{A}^{*}(\lambda)}{\mathrm{E}(\mathrm{I})}$.

Arrival rate during the busy time of the server is $\lambda \mu_{1}$ and arrival rate during vacation is $\lambda\left(\mathrm{p} \beta_{11}+\mathrm{pq} \beta_{21}\right)$.

Hence total arrival rate at the retrial queue is $\lambda\left(\mu_{1}+\mathrm{p} \beta_{11}+\right.$ $\left.\mathrm{pq} \beta_{21}\right)=\lambda \mathrm{E}(\mathrm{s})$. For stability, arrival rate should be less than the exit rate, $\lambda \mathrm{P}(\mathrm{s})<\mathrm{P}(\mathrm{I}) \frac{\mathrm{A} *(\lambda)}{\mathrm{E}(\mathrm{I})}$

This is equivalent to $\lambda\left(\mu_{1}+p \beta_{11}+p q \beta_{21}\right)<A^{*}(\lambda)$.

\section{STATIONARY DISTRIBUTION}

By supplementary variable technique, the system of steady state equations that govern the model are obtained as follows

$$
\begin{aligned}
\lambda \mathrm{I}_{0}= & (1-\mathrm{p}) \int_{0}^{\infty} \mathrm{W}_{0}(\mathrm{x}) \mu(\mathrm{x}) \mathrm{d} \mathrm{x} \\
& +(1-\mathrm{q}) \int_{0}^{\infty} \mathrm{V}_{1,0}(\mathrm{x}) \beta_{1}(\mathrm{x}) \mathrm{dx}+\int_{0}^{\infty} \mathrm{V}_{2,0}(\mathrm{x}) \beta_{2}(\mathrm{x}) \mathrm{dx} \\
\frac{\mathrm{d \textrm {I } _ { \mathrm { n } } ( \mathrm { x } )}}{\mathrm{dx}}= & -(\lambda+\eta(\mathrm{x})) \mathrm{I}_{\mathrm{n}}(\mathrm{x}), \mathrm{n} \geq 1 \\
\frac{\mathrm{dW_{0 } ( x )}}{\mathrm{dx}}= & -(\lambda+\mu(\mathrm{x})) \mathrm{W}_{0}(\mathrm{x}) \\
\frac{\mathrm{dW}_{\mathrm{n}}(\mathrm{x})}{\mathrm{dx}}= & -(\lambda+\mu(\mathrm{x})) \quad \mathrm{W}_{\mathrm{n}}(\mathrm{x})+\lambda \quad \mathrm{W}_{\mathrm{n}-1}(\mathrm{x}), \mathrm{n} \geq 1
\end{aligned}
$$

(4)

$$
\begin{aligned}
& \frac{\mathrm{dV}_{\mathrm{i}, 0}(\mathrm{x})}{\mathrm{dx}}=-\left(\lambda+\beta_{\mathrm{i}}(\mathrm{x})\right) \mathrm{V}_{\mathrm{i}, 0}(\mathrm{x}), \mathrm{i}=1,2 \\
& \frac{\mathrm{dV}_{\mathrm{i}, \mathrm{n}}(\mathrm{x})}{\mathrm{dx}}=-\left(\lambda+\beta_{\mathrm{i}}(\mathrm{x})\right) \mathrm{V}_{\mathrm{i}, \mathrm{n}}(\mathrm{x})+\lambda \mathrm{V}_{\mathrm{i}, \mathrm{n}-1}(\mathrm{x}), \mathrm{i}=1,2 ; \mathrm{n} \geq 1(6)
\end{aligned}
$$

with boundary conditions

$$
I_{n}(0)=(1-p) \int_{0}^{\infty} W_{n}(x) \mu(x) d x+(1-q) \int_{0}^{\infty} V_{1, n}(x) \beta_{1}(x) d x
$$

$$
+\int_{0}^{\infty} V_{2, n}(x) \beta_{2}(x) d x, n \geq 1
$$

$\mathrm{W}_{0}(0)=\lambda \mathrm{I}_{0}+\int_{0}^{\infty} \mathrm{I}_{1}(\mathrm{x}) \eta(\mathrm{x}) \mathrm{dx}$

$\mathrm{W}_{\mathrm{n}}(0)=\lambda \int_{0}^{\infty} \mathrm{I}_{\mathrm{n}}(\mathrm{x}) \mathrm{dx}+\int_{0}^{\infty} \mathrm{I}_{\mathrm{n}+1}(\mathrm{x}) \eta(\mathrm{x}) \mathrm{dx}, \mathrm{n} \geq 1$

$\mathrm{V}_{1, \mathrm{n}}(0)=\mathrm{p} \int_{0}^{\infty} \mathrm{W}_{\mathrm{n}}(\mathrm{x}) \mu(\mathrm{x}) \mathrm{dx}, \mathrm{n} \geq 0$

$\mathrm{V}_{2, \mathrm{n}}(0)=\mathrm{q} \int_{0}^{\infty} \mathrm{V}_{1, \mathrm{n}}(\mathrm{x}) \beta_{1}(\mathrm{x}) \mathrm{dx}, \mathrm{n} \geq 0$

The normalizing condition is $I_{0}+\sum_{n=1}^{\infty} \int_{0}^{\infty} I_{n}(x) d x+\sum_{n=0}^{\infty}[$ $\left.\int_{0}^{\infty} W_{n}(x) d x+\sum_{i=1}^{2} \int_{0}^{\infty} V_{i, n}(x) d x\right]=1$

Define $I(z, x)=\sum_{n=1}^{\infty} I_{n}(x) z^{n} ; \quad W(z, x)=\sum_{n=0}^{\infty} W_{n}(x) z^{n} \quad$ and $\mathrm{V}_{\mathrm{i}}(\mathrm{z}, \mathrm{x})=\sum_{\mathrm{n}=0}^{\infty} \mathrm{V}_{\mathrm{i}, \mathrm{n}}(\mathrm{x}) \mathrm{z}^{\mathrm{n}}, \mathrm{i}=1,2$

\section{Theorem 2}

If $\lambda\left(\mu_{1}+\mathrm{p} \beta_{11}+\mathrm{pq} \beta_{21}\right)<\mathrm{A}^{*}(\lambda)$, then the joint steady state distribution of $\{\mathrm{N}(\mathrm{t}), \mathrm{t} \geq 0\}$ under different server state are obtained as

$$
\begin{aligned}
\mathrm{I}(\mathrm{z})= & \mathrm{I}_{0} \mathrm{z}\left(1-\left[1-\mathrm{p}+\mathrm{p}(1-\mathrm{q}) \mathrm{V}_{1}^{*}(\lambda(1-\mathrm{z}))\right.\right. \\
+ & \left.\mathrm{pq} \mathrm{V}_{1}^{*}(\lambda(1-\mathrm{z})) \mathrm{V}_{2}^{*}(\lambda(1-\mathrm{z}))\right] \\
& {\left.\left[1-\mathrm{A}^{*}(\lambda)\right] \mathrm{B}^{*}(\lambda(1-\mathrm{z}))\right) / \mathrm{D}(\mathrm{z}) } \\
\mathrm{W}(\mathrm{z})= & \mathrm{I}_{0} \mathrm{~A}^{*}(\lambda)\left[1-\mathrm{B}^{*}(\lambda(1-\mathrm{z}))\right] / \mathrm{D}(\mathrm{z}) \\
\mathrm{V}_{1}(\mathrm{z})= & \mathrm{p} \mathrm{I}_{0} \mathrm{~A} *(\lambda) \mathrm{B}^{*}(\lambda(1-\mathrm{z})) \\
& {\left[1-\mathrm{V}_{1}^{*}(\lambda(1-\mathrm{z}))\right] / \mathrm{D}(\mathrm{z}) } \\
\mathrm{V}_{2}(\mathrm{z})= & \mathrm{qp} \mathrm{I}_{0} \mathrm{~A} *(\lambda) \mathrm{B} *(\lambda(1-\mathrm{z})) \mathrm{V}_{1}^{*}(\lambda(1-\mathrm{z})) \\
& {\left[1-\mathrm{V}_{2}^{*}(\lambda(1-\mathrm{z}))\right] / \mathrm{D}(\mathrm{z}) }
\end{aligned}
$$

Proof:

Multiplying equation (2) - (11) by $\mathrm{z}^{\mathrm{n}}$, summing over all possible values of $\mathrm{n}$ and solving, we get

$$
\begin{aligned}
\mathrm{I}(\mathrm{z}, \mathrm{x})= & \mathrm{I}(\mathrm{z}, 0) \mathrm{e}^{-\lambda \mathrm{x}}[1-\mathrm{A}(\mathrm{x})] \\
\mathrm{W}(\mathrm{z}, \mathrm{x})= & \mathrm{W}(\mathrm{z}, 0) \mathrm{e}^{-\lambda(1-\mathrm{z}) \mathrm{x}}[1-\mathrm{B}(\mathrm{x})] \\
\mathrm{V}_{\mathrm{i}}(\mathrm{z}, \mathrm{x})= & \mathrm{V}_{\mathrm{i}}(\mathrm{z}, 0) \mathrm{e}^{-\lambda(1-\mathrm{z}) \mathrm{x}}\left[1-\mathrm{V}_{\mathrm{i}}(\mathrm{x})\right], \mathrm{i}=1,2 \\
\mathrm{I}(\mathrm{z}, 0)= & (1-\mathrm{p}) \int_{0}^{\infty} \mathrm{W}(\mathrm{z}, \mathrm{x}) \mu(\mathrm{x}) \mathrm{dx}+(1-\mathrm{q}) \int_{0}^{\infty} \mathrm{V}_{1}(\mathrm{z}, \mathrm{x}) \beta_{1}(\mathrm{x}) \mathrm{dx} \\
& +\int_{0}^{\infty} \mathrm{V}_{2}(\mathrm{z}, \mathrm{x}) \beta_{2}(\mathrm{x}) \mathrm{dx}-\lambda \mathrm{I}_{0}
\end{aligned}
$$




$$
\begin{aligned}
\mathrm{W}(\mathrm{z}, 0) & =\lambda \mathrm{I}_{0}+\frac{\mathrm{I}(\mathrm{z}, 0)}{\mathrm{z}}\left[\mathrm{z}+(1-\mathrm{z}) \mathrm{A}^{*}(\lambda)\right] \\
\mathrm{V}_{1}(\mathrm{z}, 0) & =\mathrm{p} \int_{0}^{\infty} \mathrm{W}(\mathrm{z}, \mathrm{x}) \mu(\mathrm{x}) \mathrm{dx}=\mathrm{pW}(\mathrm{z}, 0) \mathrm{B}^{*}(\lambda(1-\mathrm{z})) \\
\mathrm{V}_{2}(\mathrm{z}, 0) & =\mathrm{q} \int_{0}^{\infty} \mathrm{V}_{1}(\mathrm{z}, \mathrm{x}) \beta_{1}(\mathrm{x}) \mathrm{dx} \\
& =\mathrm{qW}(\mathrm{z}, 0) \mathrm{B}^{*}(\lambda(1-\mathrm{z})) \mathrm{V}_{1}^{*}(\lambda(1-\mathrm{z}))
\end{aligned}
$$

Using the equations (18), (19),(22) and (23) in equation (20), we get

$$
\begin{aligned}
\mathrm{I}(\mathrm{z}, 0)= & (1-\mathrm{p}) \mathrm{W}(\mathrm{z}, 0) \mathrm{B}^{*}(\lambda(1-\mathrm{z}))+(1-\mathrm{q}) \mathrm{V}_{1}(\mathrm{z}, 0) \\
& \mathrm{V}_{1}^{*}(\lambda(1-\mathrm{z}))+\mathrm{V}_{2}(\mathrm{z}, 0) \quad \mathrm{V}_{2}^{*}(\lambda(1-\mathrm{z}))-\lambda \mathrm{I}_{0} \\
= & {\left[1-\mathrm{p}+\mathrm{p}(1-\mathrm{q}) \mathrm{V}_{1}^{*}(\lambda(1-\mathrm{z}))+\mathrm{p} \mathrm{q} \mathrm{V}_{1}^{*}(\lambda(1-\mathrm{z}))\right.} \\
& \left.\mathrm{V}_{2}^{*}(\lambda(1-\mathrm{z}))\right] \mathrm{B}^{*}(\lambda(1-\mathrm{z})) \mathrm{W}(\mathrm{z}, 0)-\lambda \mathrm{I}_{0}
\end{aligned}
$$

Substitute equation (24) in equation (21), we obtain

$$
\mathrm{W}(\mathrm{z}, 0)=\lambda \mathrm{I}_{0}(1-\mathrm{z}) \mathrm{A}^{*}(\lambda) / \mathrm{D}(\mathrm{z})
$$

where $\mathrm{D}(\mathrm{z})=\left[1-\mathrm{p}+\mathrm{p}(1-\mathrm{q}) \mathrm{V}_{1}^{*}(\lambda(1-\mathrm{z}))+\mathrm{pq} \mathrm{V}_{1}^{*}(\lambda(1-\mathrm{z}))\right.$

$$
\left.\mathrm{V}_{2}^{*}(\lambda(1-\mathrm{z}))\right] \mathrm{B} *(\lambda(1-\mathrm{z}))[\mathrm{z}+(1-\mathrm{z}) \mathrm{A} *(\lambda)]-\mathrm{z}
$$

Using the equation ( 25) in the equations (24), (22) and (23) we have the following result

$$
\begin{aligned}
\mathrm{I}(\mathrm{z}, 0)= & \left(1-\left[1-\mathrm{p}+\mathrm{p}(1-\mathrm{q}) \mathrm{V}_{1}^{*}(\lambda(1-\mathrm{z}))+\mathrm{pq} \mathrm{V}_{1}^{*}(\lambda(1-\mathrm{z}))\right.\right. \\
& \left.\left.\mathrm{V}_{2}^{*}(\lambda(1-\mathrm{z}))\right] \lambda \mathrm{I}_{0} \mathrm{z} \mathrm{B}^{*}(\lambda(1-\mathrm{z}))\right) / \mathrm{D}(\mathrm{z}) \\
\mathrm{V}_{1}(\mathrm{z}, 0)= & \mathrm{p} \lambda \mathrm{I}_{0}(1-\mathrm{z}) \mathrm{A}^{*}(\lambda) \mathrm{B}^{*}(\lambda(1-\mathrm{z})) / \mathrm{D}(\mathrm{z}) \\
\mathrm{V}_{2}(\mathrm{z}, 0)= & \mathrm{qp} \lambda \mathrm{I}_{0}(1-\mathrm{z}) \mathrm{A}^{*}(\lambda) \mathrm{B}^{*}(\lambda(1-\mathrm{z})) \\
& \mathrm{V}_{1}^{*}(\lambda(1-\mathrm{z})) / \mathrm{D}(\mathrm{z})
\end{aligned}
$$

Using equations (27) to (29) and (25) in the equations (17) (19) and integrating with respect to $\mathrm{x}$ from 0 to $\infty$ we get the equations (13) - (16) of Theorem-2.

The normalizing condition in equation (12) becomes $\mathrm{I}_{0}+$ $\mathrm{I}(1)+\mathrm{W}(1)+\mathrm{V}_{1}(1)+\mathrm{V}_{2}(1)=1$, substituting the expressions of $\mathrm{I}(1), \mathrm{W}(1), \mathrm{V}_{1}(1)$ and $\mathrm{V}_{2}(1)$ we get

$$
\mathrm{I}_{0}=\left[\mathrm{A} *(\lambda)-\lambda \mu_{1}-\mathrm{p} \lambda \beta_{11}-\mathrm{pq} \lambda \beta_{21}\right] / \mathrm{A} *(\lambda)
$$

\section{MEAN ORBIT SIZE AND MEAN SYSTEM SIZE}

The Probability generating function of the number of customers in the orbit is

$$
\begin{aligned}
\mathrm{P}_{\mathrm{q}}(\mathrm{z}) & =\mathrm{I}_{0}+\mathrm{I}(\mathrm{z})+\mathrm{W}(\mathrm{z})+\mathrm{V}_{1}(\mathrm{z})+\mathrm{V}_{2}(\mathrm{z}) \\
& =\mathrm{I}_{0}(1-\mathrm{z}) \mathrm{A} *(\lambda) / \mathrm{D}(\mathrm{z})
\end{aligned}
$$

The mean number of customer in the orbit is given by

$$
\begin{aligned}
\mathrm{L}_{\mathrm{q}} & =\lim _{\mathrm{z} \rightarrow 1} \mathrm{P}_{\mathrm{q}}^{\prime}(\mathrm{z}) \\
& =\left\{\lambda \mu_{1}[1-\mathrm{A} *(\lambda)]+\lambda \mu_{1}\left[\mathrm{p} \lambda \beta_{11}+\mathrm{pq} \lambda \beta_{21}\right]\right. \\
& +[1-\mathrm{A} *(\lambda)]\left[\mathrm{p} \lambda \beta_{11}+\mathrm{pq} \lambda \beta_{21}\right]+\mathrm{pq} \lambda^{2} \beta_{11} \beta_{21} \\
& \left.+\frac{\lambda^{2}}{2}\left[\mu_{2}+\mathrm{p} \beta_{12}+\mathrm{pq} \beta_{22}\right]\right\} / \\
& \left\{\mathrm{A} *(\lambda)-\lambda \mu_{1}-\mathrm{p} \lambda \beta_{11}-\mathrm{pq} \lambda \beta_{21}\right\}
\end{aligned}
$$

The Probability generating function of the number of customer in the system is

$$
\begin{aligned}
\mathrm{P}_{\mathrm{s}}(\mathrm{z}) & =\mathrm{I}_{0}+\mathrm{I}(\mathrm{z})+\mathrm{z} \mathrm{W}(\mathrm{z})+\mathrm{V}_{1}(\mathrm{z})+\mathrm{V}_{2}(\mathrm{z}) \\
& =\mathrm{I}_{0}(1-\mathrm{z}) \mathrm{A}^{*}(\lambda) \mathrm{B}^{*}(\lambda(1-\mathrm{z})) / \mathrm{D}(\mathrm{z})
\end{aligned}
$$

The mean number of customer in the system is given by

$$
\begin{aligned}
\mathrm{L}_{\mathrm{s}} & =\lim _{\mathrm{z} \rightarrow 1} \mathrm{P}_{\mathrm{s}}^{\prime}(\mathrm{z}) \\
& =\lambda \mu_{1}+\mathrm{L}_{\mathrm{q}}
\end{aligned}
$$

\section{PERFORMANCE MEASURES}

Performance measures for the system under steady state condition are given below

- The steady state probability that the server is idle in the empty system is

$$
\mathrm{I}_{0}=\left[\mathrm{A} *(\lambda)-\lambda \mu_{1}-\mathrm{p} \lambda \beta_{11}-\mathrm{pq} \lambda \beta_{21}\right] / \mathrm{A} *(\lambda)
$$

- The steady state probability that the server is idle in the non - empty system is

$$
\mathrm{I}_{1}=[1-\mathrm{A} *(\lambda)]\left[\lambda \mu_{1}+\mathrm{p} \lambda \beta_{11}+\mathrm{pq} \lambda \beta_{21}\right] / \mathrm{A} *(\lambda)
$$

- The steady state probability that the server is busy is $\mathrm{W}=\lambda \mu_{1}$

- The steady state probability that the server is on phase 1 vacation is

$$
\mathrm{V}_{1}=\mathrm{p} \lambda \beta_{11}
$$

- The steady state probability that the server is on phase 2 vacation is

$$
\mathrm{V}_{2}=\operatorname{pq} \lambda \beta_{21}
$$

- The steady state probability that the server is on vacation is

$\mathrm{V}=\mathrm{V}_{1}+\mathrm{V}_{2}=\mathrm{p} \lambda \beta_{11}+\mathrm{pq} \lambda \beta_{21}$

\section{SPECIAL CASES}

\section{Case 1}

If $q=1$, the results for $M / G / 1$ retrial queue with 2 phase heterogeneous vacations are obtained. In this case

$$
\begin{aligned}
& \mathrm{I}_{0}=\left[\mathrm{A} *(\lambda)-\lambda \mu_{1}-\mathrm{p} \lambda \beta_{11}-\mathrm{p} \lambda \beta_{21}\right] / \mathrm{A} *(\lambda) \\
& \mathrm{I}_{1}=[1-\mathrm{A} *(\lambda)]\left[\lambda \mu_{1}+\mathrm{p} \lambda \beta_{11}+\mathrm{p} \lambda \beta_{21}\right] / \mathrm{A} *(\lambda) \\
& \mathrm{W}=\lambda \mu_{1}
\end{aligned}
$$




$$
\begin{aligned}
& \mathrm{V}= \mathrm{p} \lambda \beta_{11}+\mathrm{p} \lambda \beta_{21} \\
& \mathrm{~L}_{\mathrm{q}}=\left\{\lambda \mu_{1}[1-\mathrm{A} *(\lambda)]+\lambda \mu_{1}\left[\mathrm{p} \lambda \beta_{11}+\mathrm{p} \lambda \beta_{21}\right]\right. \\
&+\left[1-\mathrm{A}^{*}(\lambda)\right]\left[\mathrm{p} \lambda \beta_{11}+\mathrm{p} \lambda \beta_{21}\right]+\mathrm{p} \lambda^{2} \beta_{11} \beta_{21}+ \\
&\left.\frac{\lambda^{2}}{2}\left[\mu_{2}+\mathrm{p} \beta_{12}+\mathrm{p} \beta_{22}\right]\right\} /\left\{\mathrm{A}^{*}(\lambda)-\lambda \mu_{1}-\mathrm{p} \lambda \beta_{11}-\mathrm{p} \lambda \beta_{21}\right\} \\
& \mathrm{L}_{\mathrm{s}}=\lambda \mu_{1}+\mathrm{L}_{\mathrm{q}}
\end{aligned}
$$

\section{Case 2}

If we take vacation rates of phase 1 and phase 2 are equal then we get results of $\mathrm{M} / \mathrm{G} / 1$ retrial queue with Erlangian -2 vacations.

$$
\begin{aligned}
\mathrm{I}_{0}= & {\left[\mathrm{A} *(\lambda)-\lambda \mu_{1}-\mathrm{p} \lambda \beta_{1}-\mathrm{pq} \lambda \beta_{1}\right] / \mathrm{A}^{*}(\lambda) } \\
\mathrm{I}_{1}= & {[1-\mathrm{A} *(\lambda)]\left[\lambda \mu_{1}+\mathrm{p} \lambda \beta_{1}+\mathrm{pq} \lambda \beta_{1}\right] / \mathrm{A} *(\lambda) } \\
\mathrm{W}= & \lambda \mu_{1} \\
\mathrm{~V}= & \mathrm{p} \lambda \beta_{1}+\mathrm{pq} \lambda \beta_{1} \\
\mathrm{~L}_{\mathrm{q}}= & \left\{\lambda \mu_{1}[1-\mathrm{A} *(\lambda)]+\lambda \mu_{1}\left[\mathrm{p} \lambda \beta_{1}+\mathrm{pq} \lambda \beta_{1}\right]+[1-\mathrm{A} *(\lambda)]\right. \\
& {\left.\left[\mathrm{p} \lambda \beta_{1}+\mathrm{pq} \lambda \beta_{1}\right]+\mathrm{pq} \lambda^{2} \beta_{1}^{2}+\frac{\lambda^{2}}{2}\left[\mu_{2}+\mathrm{p} \beta_{1}+\mathrm{pq} \beta_{2}\right]\right\} / } \\
& \left\{\mathrm{A} *(\lambda)-\lambda \mu_{1}-\mathrm{p} \lambda \beta_{1}-\mathrm{pq} \lambda \beta_{1}\right\} \\
\mathrm{L}_{\mathrm{s}}= & \lambda \mu_{1}+\mathrm{L}_{\mathrm{q}}
\end{aligned}
$$

Case 3

If $\mathrm{q}=0$, then we the results of this mode coincide with the results of M/G/1 retrial queue with vacation by Maragatha Sundari, S., and Srinivasan, S.,[8].

\section{Case 4}

If $p=0$, then we get the fundamental retrial queueing model without vacations.

\section{Case 5}

If $A^{*}(\lambda) \rightarrow 1$, then we get the results for $\mathrm{M} / \mathrm{G} / 1$ queueing system with Bernoulli and phase type vacation.

$$
\begin{aligned}
\mathrm{I}_{0}= & {\left[1-\lambda \mu_{1}-\mathrm{p} \lambda \beta_{11}-\mathrm{pq} \lambda \beta_{21}\right] } \\
\mathrm{W}= & \lambda \mu_{1} \\
\mathrm{~V}= & \mathrm{p} \lambda \beta_{11}+\mathrm{pq} \lambda \beta_{21} \\
\mathrm{~L}_{\mathrm{q}}= & \left\{\lambda \mu_{1}\left[\mathrm{p} \lambda \beta_{11}+\mathrm{pq} \lambda \beta_{21}\right]+\mathrm{pq} \lambda^{2} \beta_{11} \beta_{21}+\frac{\lambda^{2}}{2}\right. \\
& {\left.\left[\mu_{2}+\mathrm{p} \beta_{12}+\mathrm{pq} \beta_{22}\right]\right\} /\left\{1-\lambda \mu_{1}-\mathrm{p} \lambda \beta_{11}-\mathrm{pq} \lambda \beta_{21}\right\} } \\
\mathrm{L}_{\mathrm{s}}= & \lambda \mu_{1}+\mathrm{L}_{\mathrm{q}}
\end{aligned}
$$

\section{Case 6}

Assume that $A^{*}(\lambda) \rightarrow 1$, service time is deterministic of length $d$ and vacation time are exponential with rate $\beta_{1}$ and $\beta_{2}$ respectively for phase 1 and phase 2 . Then the results coincide with Jehad Al- Jararha and Madan [6].

\section{NUMERICAL STUDY}

In order to verify the efficiency of our analytical results, we perform numerical experiments by using MATLAB. Assume that retrial time, service time, phase 1 vacation time, phase 2 vacation time are exponentially distributed with the rate $\eta, \mu$, $\beta_{1}, \beta_{2}$ respectively.

The fig $1-4$ shows the effect of $\lambda$ on $\left(\mu, I_{0}\right),\left(\mu, W_{1}\right),\left(\mu, I_{1}\right)$ and $\left(\mu, L_{S}\right)$ for fixed $\left(\eta, p, q, \beta_{1}, \beta_{2}\right)=(20,0.5,0.2,8,10)$ Table 1 displays the performance measures for $\left(\lambda, \eta, \mu, \beta_{1}, \beta_{2}\right)$ $=(3,20,20,7,12)$ and for the various values of $p$ and $q$ from 0 to 1 . As expected, for increasing values of $p$ and $q$ the performance measures $I_{1}, V_{1}, V_{2}, L_{s}$ increase and $I_{0}$ decreases. Table 2 shows the results for the various values of $\eta, \mu$ with the parameter values $\left(\lambda, p, q, \beta_{1}, \beta_{2}\right)=(4,0.5,0.2,6,8)$. As expected, $\mathrm{W}_{1}$ is constant for all $\mathrm{p}$ and $\mathrm{q}$ and $\mathrm{V}_{1}$ and $\mathrm{V}_{2}$ are constant for all $\eta$ and $\mu$.

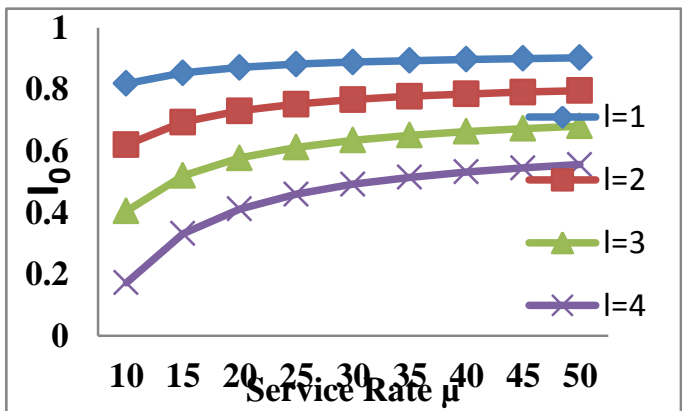

Fig 1

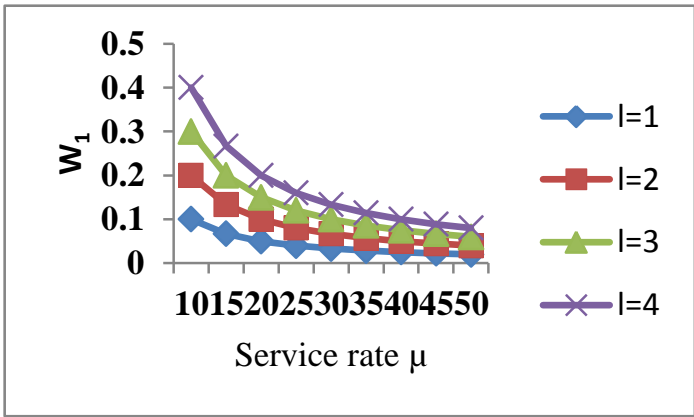

Fig 2

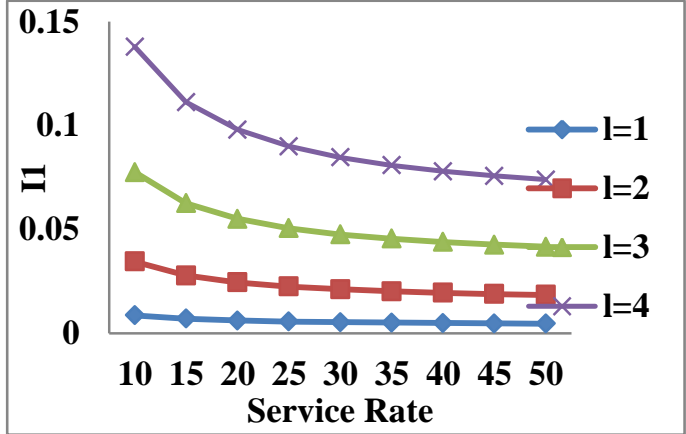

Fig 3 


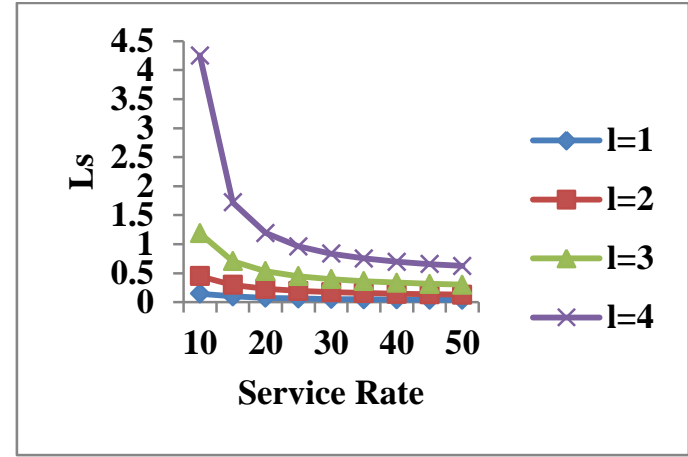

Fig 4

Table 1. Performance measures for the varying values of $p$ and $q$

\begin{tabular}{|c|c|c|c|c|c|c|c|}
\hline p & $\mathbf{q}$ & $\mathbf{I}_{\mathbf{0}}$ & $\mathbf{I}_{1}$ & $W_{1}$ & $V_{11}$ & $\mathbf{V}_{21}$ & $\mathbf{L}_{\mathrm{s}}$ \\
\hline \multirow{6}{*}{0} & 0 & 0.828 & 0.023 & 0.150 & 0.000 & 0.000 & 0.209 \\
\hline & 0.2 & 0.828 & 0.023 & 0.150 & 0.000 & 0.000 & 0.209 \\
\hline & 0.4 & 0.828 & 0.023 & 0.150 & 0.000 & 0.000 & 0.209 \\
\hline & 0.6 & 0.828 & 0.023 & 0.150 & 0.000 & 0.000 & 0.209 \\
\hline & 0.8 & 0.828 & 0.023 & 0.150 & 0.000 & 0.000 & 0.209 \\
\hline & 1 & 0.828 & 0.023 & 0.150 & 0.000 & 0.000 & 0.209 \\
\hline \multirow{6}{*}{0.2} & 0 & 0.729 & 0.035 & 0.150 & 0.086 & 0.000 & 0.312 \\
\hline & 0.2 & 0.717 & 0.037 & 0.150 & 0.086 & 0.010 & 0.330 \\
\hline & 0.4 & 0.706 & 0.038 & 0.150 & 0.086 & 0.020 & 0.349 \\
\hline & 0.6 & 0.694 & 0.040 & 0.150 & 0.086 & 0.030 & 0.368 \\
\hline & 0.8 & 0.683 & 0.041 & 0.150 & 0.086 & 0.040 & 0.388 \\
\hline & 1 & 0.671 & 0.043 & 0.150 & 0.086 & 0.050 & 0.408 \\
\hline \multirow{6}{*}{0.4} & 0 & 0.630 & 0.048 & 0.150 & 0.171 & 0.000 & 0.449 \\
\hline & 0.2 & 0.607 & 0.051 & 0.150 & 0.171 & 0.020 & 0.496 \\
\hline & 0.4 & 0.584 & 0.054 & 0.150 & 0.171 & 0.040 & 0.548 \\
\hline & 0.6 & 0.561 & 0.057 & 0.150 & 0.171 & 0.060 & 0.603 \\
\hline & 0.8 & 0.538 & 0.060 & 0.150 & 0.171 & 0.080 & 0.663 \\
\hline & 1 & 0.515 & 0.063 & 0.150 & 0.171 & 0.100 & 0.729 \\
\hline \multirow{6}{*}{0.6} & 0 & 0.532 & 0.061 & 0.150 & 0.257 & 0.000 & 0.635 \\
\hline & 0.2 & 0.497 & 0.066 & 0.150 & 0.257 & 0.030 & 0.735 \\
\hline & 0.4 & 0.463 & 0.070 & 0.150 & 0.257 & 0.060 & 0.851 \\
\hline & 0.6 & 0.428 & 0.075 & 0.150 & 0.257 & 0.090 & 0.984 \\
\hline & 0.8 & 0.394 & 0.079 & 0.150 & 0.257 & 0.120 & 1.141 \\
\hline & 1 & 0.359 & 0.084 & 0.150 & 0.257 & 0.150 & 1.329 \\
\hline \multirow{4}{*}{0.8} & 0 & 0.433 & 0.074 & 0.150 & 0.343 & 0.000 & 0.907 \\
\hline & 0.2 & 0.387 & 0.080 & 0.150 & 0.343 & 0.040 & 1.111 \\
\hline & 0.4 & 0.341 & 0.086 & 0.150 & 0.343 & 0.080 & 1.370 \\
\hline & 0.6 & 0.295 & 0.092 & 0.150 & 0.343 & 0.120 & 1.709 \\
\hline
\end{tabular}

\begin{tabular}{|c|c|c|c|c|c|c|c|}
\hline \multirow{1}{*}{} & 0.8 & 0.249 & 0.098 & 0.150 & 0.343 & 0.160 & 2.174 \\
\hline \multirow{4}{*}{1} & 1 & 0.203 & 0.104 & 0.150 & 0.343 & 0.200 & 2.849 \\
\hline \multirow{4}{*}{1} & 0 & 0.335 & 0.087 & 0.150 & 0.429 & 0.000 & 1.339 \\
\cline { 2 - 8 } & 0.2 & 0.277 & 0.094 & 0.150 & 0.429 & 0.050 & 1.784 \\
\cline { 2 - 8 } & 0.4 & 0.220 & 0.102 & 0.150 & 0.429 & 0.100 & 2.463 \\
\cline { 2 - 8 } & 0.6 & 0.162 & 0.109 & 0.150 & 0.429 & 0.150 & 3.624 \\
\cline { 2 - 8 } & 0.8 & 0.105 & 0.117 & 0.150 & 0.429 & 0.200 & 6.060 \\
\hline & 1 & 0.047 & 0.124 & 0.150 & 0.429 & 0.250 & 14.437 \\
\hline
\end{tabular}

Table 2. Performance measures for the varying values of $\eta$ and $\mu$

\begin{tabular}{|c|c|c|c|c|c|c|c|}
\hline$\eta$ & $\boldsymbol{\mu}$ & $\mathbf{I}_{0}$ & $I_{1}$ & $\mathbf{W}$ & $V_{1}$ & $V_{2}$ & $\mathbf{L}_{\mathrm{s}}$ \\
\hline \multirow{6}{*}{5} & 25 & 0.022 & 0.435 & 0.160 & 0.333 & 0.050 & 49.825 \\
\hline & 30 & 0.070 & 0.413 & 0.133 & 0.333 & 0.050 & 14.891 \\
\hline & 35 & 0.104 & 0.398 & 0.114 & 0.333 & 0.050 & 9.641 \\
\hline & 40 & 0.130 & 0.387 & 0.100 & 0.333 & 0.050 & 7.528 \\
\hline & 45 & 0.150 & 0.378 & 0.089 & 0.333 & 0.050 & 6.389 \\
\hline & 50 & 0.166 & 0.371 & 0.080 & 0.333 & 0.050 & 5.677 \\
\hline \multirow{6}{*}{10} & 25 & 0.239 & 0.217 & 0.160 & 0.333 & 0.050 & 3.058 \\
\hline & 30 & 0.277 & 0.207 & 0.133 & 0.333 & 0.050 & 2.515 \\
\hline & 35 & 0.303 & 0.199 & 0.114 & 0.333 & 0.050 & 2.214 \\
\hline & 40 & 0.323 & 0.193 & 0.100 & 0.333 & 0.050 & 2.022 \\
\hline & 45 & 0.339 & 0.189 & 0.089 & 0.333 & 0.050 & 1.890 \\
\hline & 50 & 0.351 & 0.185 & 0.080 & 0.333 & 0.050 & 1.793 \\
\hline \multirow{6}{*}{15} & 25 & 0.312 & 0.145 & 0.160 & 0.333 & 0.050 & 1.958 \\
\hline & 30 & 0.346 & 0.138 & 0.133 & 0.333 & 0.050 & 1.680 \\
\hline & 35 & 0.370 & 0.133 & 0.114 & 0.333 & 0.050 & 1.515 \\
\hline & 40 & 0.388 & 0.129 & 0.100 & 0.333 & 0.050 & 1.407 \\
\hline & 45 & 0.402 & 0.126 & 0.089 & 0.333 & 0.050 & 1.330 \\
\hline & 50 & 0.413 & 0.124 & 0.080 & 0.333 & 0.050 & 1.273 \\
\hline \multirow{6}{*}{20} & 25 & 0.348 & 0.109 & 0.160 & 0.333 & 0.050 & 1.580 \\
\hline & 30 & 0.380 & 0.103 & 0.133 & 0.333 & 0.050 & 1.375 \\
\hline & 35 & 0.403 & 0.100 & 0.114 & 0.333 & 0.050 & 1.252 \\
\hline & 40 & 0.420 & 0.097 & 0.100 & 0.333 & 0.050 & 1.170 \\
\hline & 45 & 0.433 & 0.094 & 0.089 & 0.333 & 0.050 & 1.111 \\
\hline & 50 & 0.444 & 0.093 & 0.080 & 0.333 & 0.050 & 1.067 \\
\hline \multirow{6}{*}{25} & 25 & 0.370 & 0.087 & 0.160 & 0.333 & 0.050 & 1.388 \\
\hline & 30 & 0.401 & 0.083 & 0.133 & 0.333 & 0.050 & 1.218 \\
\hline & 35 & 0.423 & 0.080 & 0.114 & 0.333 & 0.050 & 1.114 \\
\hline & 40 & 0.439 & 0.077 & 0.100 & 0.333 & 0.050 & 1.044 \\
\hline & 45 & 0.452 & 0.076 & 0.089 & 0.333 & 0.050 & 0.994 \\
\hline & 50 & 0.463 & 0.074 & 0.080 & 0.333 & 0.050 & 0.957 \\
\hline \multirow{6}{*}{30} & 25 & 0.384 & 0.072 & 0.160 & 0.333 & 0.050 & 1.273 \\
\hline & 30 & 0.414 & 0.069 & 0.133 & 0.333 & 0.050 & 1.122 \\
\hline & 35 & 0.436 & 0.066 & 0.114 & 0.333 & 0.050 & 1.029 \\
\hline & 40 & 0.452 & 0.064 & 0.100 & 0.333 & 0.050 & 0.967 \\
\hline & 45 & 0.465 & 0.063 & 0.089 & 0.333 & 0.050 & 0.922 \\
\hline & 50 & 0.475 & 0.062 & 0.080 & 0.333 & 0.050 & 0.888 \\
\hline \multirow{2}{*}{35} & 25 & 0.395 & 0.062 & 0.160 & 0.333 & 0.050 & 1.195 \\
\hline & 30 & 0.424 & 0.059 & 0.133 & 0.333 & 0.050 & 1.057 \\
\hline
\end{tabular}




\begin{tabular}{|c|c|c|c|c|c|c|c|}
\hline \multirow{6}{*}{35} & 0.446 & 0.057 & 0.114 & 0.333 & 0.050 & 0.972 \\
\hline 40 & 0.461 & 0.055 & 0.100 & 0.333 & 0.050 & 0.914 \\
\hline 45 & 0.474 & 0.054 & 0.089 & 0.333 & 0.050 & 0.872 \\
\hline 50 & 0.484 & 0.053 & 0.080 & 0.333 & 0.050 & 0.841 \\
\hline \multirow{6}{*}{40} & 25 & 0.402 & 0.054 & 0.160 & 0.333 & 0.050 & 1.140 \\
\hline & 30 & 0.432 & 0.052 & 0.133 & 0.333 & 0.050 & 1.010 \\
\cline { 2 - 8 } & 35 & 0.453 & 0.050 & 0.114 & 0.333 & 0.050 & 0.930 \\
\hline & 40 & 0.468 & 0.048 & 0.100 & 0.333 & 0.050 & 0.876 \\
\hline & 45 & 0.481 & 0.047 & 0.089 & 0.333 & 0.050 & 0.837 \\
\hline
\end{tabular}

\section{CONCLUSION}

In this paper, a single server retrial queue with Bernoulli vacation and phase type vacation is analyzed under the condition of stability. Several system performance measures are computed in steady state. Some numerical illustrations are also presented.

\section{ACKNOWLEDGEMENTS}

The authors are very grateful to the referees for their valuable suggestions to improve this paper in the present form.

\section{REFERENCES}

[1] Amar Aissani. 2011. An M/G/1 Energetic Retrial Queue with Vacations and Control. IMA Journal of Management Mathematics. 22:13-32.

[2] Arivudainambi, D. and Godhandaraman, P.2012. A Batch Arrival Retrial Queue with Two Phase of Service, Feedback and $\mathrm{K}$ Optional Vacations. Applied Mathematical Sciences. 6; 22;1071-1087.

[3] Badamchi Zadeh, A. 2012. A Batch Arrival Queue System with Coxian -2 Server Vacations and Admissibility Restricted.American Journal of Industrial and Business Management.2;47-54.
[4] Choudhury, G. and Jau- ChuanKe.2012. A batch Arrival Retrial Queue with General Retrial Times Under Bernoulli Vacation Schedule for Unreliable Server and Delaying Repair. Applied Mathematical Modelling. 36;255-269.

[5] Ebenesar Anna Bagyam, J. and Udaya Chandrika, K.2010. Single server Retrial Queueing System with Two Different Vacation Policies. International Journal of Contemporary Mathematical Sciences.5; ;32;1591-1598.

[6] Jehad Al-Jararha and Madan K.2002. Steady State Analysis of an M/D/1 Queue with Coxian-2 Server Vacations and a Single Vacation Policy. Information and Management Sciences.13;4; 69-81.

[7] Jia, S., Chen,Y. and Liu, J.2011. Steady State Analysis of Bernoulli Feedback on Geom X/g/1 Queue with Multiple Vacation and Setup Times. International Journal of Applied Physics and Mathematics.1;1.

[8] Maragatha Sundari, S. and Srinivasan, S.2012. Analysis of Transient Behaviour of M/G/1 Queue with Single Vacation. International Journal of Pure and Applied Mathematics. 76;1;149-156.

[9] Muthu Ganapathi, Ayyappan, and Gopal Sekar. 2011.Study of Multi Server retrial Queueing System Under Vacation Policies By Direct Truncation Method. QTNA. 23-26.

[10] Purohit, G.N., Madhu Jain and Shinu Rani. 2012. M/M/1 Retrial Queue with Constant Retrial Policy, Unreliable Sever, Threshold based Recovery and State dependent Arrival Rates. Applied Mathematical Sciences.6;37;1837 -1846 .

[11] Tuan PhungDuc and Kenichi Kawanishi. 2011. Multi Server Retrial Queue with After Call Work. Numerical Algebra, Control and Optimization. 1;4; 639-656.

[12] Wang, W. and Xu, X.2011.Performance Analysis of GI/Geom/1 with Single Working Vacation and Setup Times. Journal of Information and Computational Science. 8;14; 3083, 3090. 